BRAZIULIAN JOURNAL

OF MEDICAL AND BIOLOGICAL RESFARCH

www.bjournal.com.br
ISSN 0100-879X

Volume 44 (11) 1070-1193 November 2011

BIOMEDICAL SCIENCES

AND

CLINICAL INVESTIGATION

Braz J Med Biol Res, November 2011, Volume 44(11) 1125-1133

doi: 10.1590/S0100-879X2011007500130

The cytotoxicity of methacryloxylethyl cetyl ammonium chloride, a cationic antibacterial monomer, is related to oxidative stress and the intrinsic mitochondrial apoptotic pathway

Ma Sai, Shan Le-qun, Xiao Yu-hong, Li Fang, Huang Li, Lijuan Shen and Chen Ji-hua

The Brazilian Journal of Medical and Biological Research is partially financed by

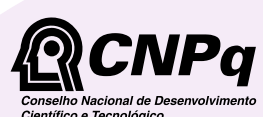

da Ciência e Tecnologia

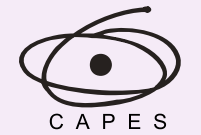

Ministério da Educação
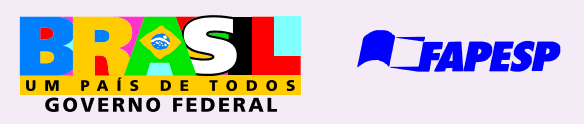

Institutional Sponsors
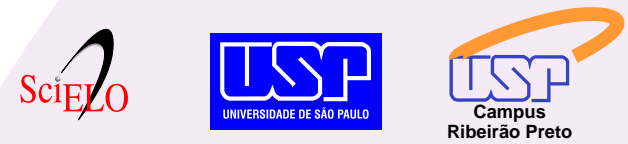

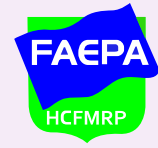

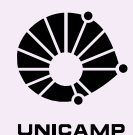

UNICAMP

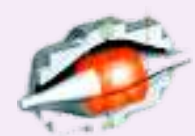

SHIMADZU

Explore High - Performance MS Orbitrap Technology In Proteomics \& Metabolomics

analitica Thermo 


\title{
The cytotoxicity of methacryloxylethyl cetyl ammonium chloride, a cationic antibacterial monomer, is related to oxidative stress and the intrinsic mitochondrial apoptotic pathway
}

\author{
Ma Sai ${ }^{1 *}$, Shan Le-qun ${ }^{2 *}$, Xiao Yu-hong ${ }^{3}$, Li Fang1 ${ }^{1}$, Huang Li ${ }^{1}$ \\ Lijuan Shen ${ }^{1}$ and Chen Ji-hua ${ }^{1}$ \\ 1Department of Prosthodontics, School of Stomatology, ${ }^{2}$ Department of Orthopedics, \\ Tangdu Hospital, Fourth Military Medical University, Xi'an, China \\ ${ }^{3}$ Department of Stomatology, Kunming General Hospital of PLA, Kunming, China
}

\begin{abstract}
Antibacterial monomers incorporated in dentin bonding systems may have toxic effects on the pulp. Thus, the cytotoxicity of antibacterial monomers and its underlying mechanisms must be elucidated to improve the safety of antibacterial monomer application. The influence of an antibacterial monomer, methacryloxylethyl cetyl ammonium chloride (DMAE-CB), on the vitality of L929 mouse fibroblasts was tested using MTT assay. Cell cycle progression was studied using flow cytometry. Production of intracellular reactive oxygen species (ROS) after DMAE-CB treatment was measured using 2,7-dichlorodihydrofluorescein diacetate staining and flow cytometry analysis. Loss of mitochondrial membrane potential, disturbance of Bcl-2 and Bax expression, as well as release of cytochrome $\mathrm{C}$ were also measured using flow cytometry analysis or Western blot to explore the possible involvement of the mitochondrial-related apoptotic pathway. DMAE-CB elicited cell death in a dose-dependent manner and more than $50 \%$ of cells were killed after treatment with $30 \mu \mathrm{M}$ of the monomer. Both necrosis and apoptosis were observed. DMAE-CB also induced G1- and G2-phase arrest. Increased levels of intracellular ROS were observed after $1 \mathrm{~h}$ and this overproduction was further enhanced by 6-h treatment with the monomer. DMAE-CB may cause apoptosis by disturbing the expression of $\mathrm{Bcl}-2$ and Bax, reducing the mitochondrial potential and inducing release of cytochrome C. Taken together, these findings suggest that the toxicity of the antibacterial monomer DMAE-CB is associated with ROS production, mitochondrial dysfunction, cell cycle disturbance, and cell apoptosis/necrosis.
\end{abstract}

Key words: Quaternary ammonium compounds; Dental monomer; Cytotoxicity; Apoptosis; Reactive oxygen species; Methacryloxylethyl cetyl ammonium chloride

\section{Introduction}

The development of resin-based adhesives has permitted more satisfactory restorative treatment with good esthetic appearance. Despite the simplified procedure of their clinical use and improved immediate bonding effectiveness, contemporary adhesive restoratives suffer from a major shortcoming of limited durability in vivo (1). Microleakage along the dentin-resin interface and subsequent bacterial invasion represent a primary cause of reduced bonding durability. Therefore, development of "bioactive" bonding materials with antibacterial activity is recognized as an important dimension of future development (2). A polymerizable antibacterial monomer may be an ideal candidate for bioactive resin-based material. Before polymerization, free antibacterial monomers can eradicate bacteria like a disinfectant, while after polymerization the immobilized antibacterial component can keep on inhibiting the secondary invasion of bacteria along the bonded interface. There have been various attempts to develop antibacterial mono-

Correspondence: Chen Ji-hua, Department of Prosthodontics, School of Stomatology, Fourth Military Medical University, Xi'an, 710032 China. Fax: +86-029-8477-6329. E-mail: jhchen@fmmu.edu.cn

*These authors contributed equally to this study.

Received April 30, 2011. Accepted September 16, 2011. Available online October 14, 2011. Published November 14, 2011. 
mers, and up to now several such monomers have been reported, including methacryloyloxydodecylpyridinium bromide (MDPB) from Imazato's group and methacryloxylethyl cetyl ammonium chloride (DMAE-CB) from Chen's group. Actually, the incorporation of DMAE-CB into dental adhesives has proved as an effective strategy to achieve bioactive bonding with reliable bacterial inhibitory effect and satisfactory bonding ability (3-5). However, the biological safety of the antibacterial monomer DMAE-CB, which is of vital importance for clinical success, has not yet been thoroughly studied.

The concern for the biological safety of antibacterial monomers is highlighted by the fact that major monomers and co-monomers of commonly used resin-based dental materials have been identified as cytotoxic compounds (6). Among various dental monomers, 2-hydroxyethyl methacrylate (HEMA) and triethylene glycol dimethacrylate (TEGDMA) have attracted the most attention because these hydrophilic monomers have been detected in the eluent of dental adhesives (7), and have proved to be able to reach sufficient concentrations to cause cellular damage after diffusing through dentin (8). Although the molecular mechanisms underlying the genetic and cellular toxicity of dental monomers remain to be elucidated, recent investigations have indicated that the disturbance of cellular redox balance may be an important contributor to monomer-induced DNA damage, cell-cycle arrest and eventually apoptosis in various cell types (6). However, as is the case for antibacterial monomers, limited data have been reported about their cytotoxicity and related mechanisms. In a recent paper, Imazato et al. (9) reported that, like other dental monomers, the antibacterial monomer MDPB exhibited a significant inhibitory effect on the proliferation, differentiation and mineralization of odontoblast-like cells. But the question of whether the cytotoxicity of antibacterial monomers is also related to oxidative stress is still unanswered.

Therefore, it was the objective of this investigation to thoroughly investigate the cytotoxicity of the antibacterial monomer DMAE-CB and explore the possible involvement of oxidative stress and the mitochondrial apoptotic pathway in the cytotoxic effect of this antibacterial monomer by using L929 mouse fibroblasts.

\section{Material and Methods}

\section{Cell culture}

L929 mouse fibroblasts were cultured with RPMI 1640 (Invitrogen, USA) supplemented with 10\% fetal bovine serum (FBS; Invitrogen), $100 \mathrm{U} / \mathrm{mL}$ penicillin and $100 \mathrm{mg} /$ $\mathrm{mL}$ streptomycin. The cells were maintained in a humidified $5 \% \mathrm{CO}_{2}$ balanced-air incubator at $37^{\circ} \mathrm{C}$.

\section{Influence of DMAE-CB on cell proliferation: MTT assay}

The antibacterial monomer DMAE-CB was dissolved in complete culture medium containing dimethyl sulfoxide (DMSO; Amresco, USA) to various concentrations ranging from 5 to $50 \mu \mathrm{M}$. The final DMSO concentration $(0.25 \%$, $\mathrm{v} / \mathrm{v}$ ) was identical for all DMAE-CB solutions and was non-toxic to L929 cultures (data not shown).

The influence of DMAE-CB on the proliferation of L929 cells was studied using the MTT assay. Cells were seeded in 96-well plates at the density of $5 \times 10^{3}$ cells/well and incubated at $37^{\circ} \mathrm{C}$ under $5 \% \mathrm{CO}_{2}$ for approximately $24 \mathrm{~h}$. After growing to $80 \%$ confluence, the cells were treated with various DMAE-CB concentrations for $24 \mathrm{~h}$. Then, $20 \mu \mathrm{L} 5 \mathrm{mg} / \mathrm{mL}$ MTT (sterilized with a $0.22-\mu \mathrm{m}$ syringe filter; Sigma-Aldrich, USA) was added to each well and the cells were incubated for a further $4 \mathrm{~h}$. After disposal of the culture medium and the addition of $200 \mu \mathrm{L}$ DMSO, the absorbance of each well was measured at $492 \mathrm{~nm}$ spectrophotometrically with a MultiskanAscent V1.24 spectrometer (Thermo Fisher Scientific Inc., USA).

\section{Influence of DMAE-CB on cell cycle progression: PI staining and flow cytometry analysis \\ Cells were seeded in 6-well plates at the density of 1} $\mathrm{x} 10^{5} / \mathrm{mL}$, incubated for $24 \mathrm{~h}$ and then exposed to various concentrations of DMAE-CB for $24 \mathrm{~h}$. Following exposure, both floating and attached cells were harvested, washed twice with PBS and resuspended. After fixing with $10 \mathrm{~mL}$ cold $75 \%$ ethanol at $4^{\circ} \mathrm{C}$ overnight, the cells were washed with PBS and subsequently stained with $200 \mu \mathrm{L}$ propidium iodide (PI; Sigma-Aldrich) at room temperature for 20$30 \mathrm{~min}$. Cell cycle distribution was determined by flow cytometry (Becton-Dickinson FACScan, USA). A total of 5000 cells were analyzed for each sample.

\section{Induction of apoptosis by DMAE-CB: annexin V-FITC staining and flow cytometry analysis}

Cells were seeded in 6-well plates at the density of $1 \times 10^{5} / \mathrm{mL}$ and incubated for $24 \mathrm{~h}$ and then exposed to various concentrations of DMAE-CB for $24 \mathrm{~h}$. Following exposure, both floating and attached cells were collected, washed twice with PBS and then stained with annexin V-FITC (BD PharMingen, USA) and PI for 10 min at $4^{\circ} \mathrm{C}$ according to a standard protocol. Early apoptotic, late apoptotic/necrotic, necrotic and viable cells were determined by flow cytometry analysis (Becton-Dickinson FACScan).

\section{Influence of DMAE-CB on intracellular ROS production:} DCFH-DA staining and flow cytometry analysis

L929 cells $\left(1 \times 10^{5} / \mathrm{mL}\right)$ were seeded in 6-well plates. After $24 \mathrm{~h}$, cells were incubated with fresh medium containing DMSO $(0.25 \%, \mathrm{v} / \mathrm{v})$ or various concentrations of DMAE-CB for another 1 or $6 \mathrm{~h}$. To measure the production of reactive oxygen species (ROS) after DMAE-CB treatment, the cells were then stained with $10 \mu \mathrm{M}$ DCFH-DA (2,7-dichlorofluorescein diacetate; Beyotime, China) for 
$20 \mathrm{~min}$ at $37^{\circ} \mathrm{C}$, detached, washed with culture medium containing no FBS, and immediately subjected to flow cytometry analysis (Becton-Dickinson FACScan).

\section{Influence of DMAE-CB on mitochondrial membrane potential (MMP): DiOC6 staining and flow cytometry analysis}

The loss of mitochondrial membrane potential in L929 cells was measured by flow cytometry with a fluorescent dye that accumulates in mitochondria as a function of membrane potential (3,3-dihexyloxacarbocyanine iodide, DiOC6; Invitrogen). L929 cells were treated with different concentrations of DMAE-CB for $12 \mathrm{~h}$ before harvesting, stained with the dye for $15 \mathrm{~min}$ at $37^{\circ} \mathrm{C}$, and analyzed by flow cytometry (Becton-Dickinson FACScan).

Influence of DMAE-CB on Bcl-2 and Bax expression and cytochrome $C$ release: Western blot analysis

Cells were treated with $20 \mu \mathrm{M}$ DMAE-CB for 1,6 , or $12 \mathrm{~h}$. The cells were then collected, washed with cold PBS, and lysed with lysis buffer (1 M Tris-HCA, $5 \mathrm{M} \mathrm{NaCl}$, $1 \%$ Nonidet P-40 (v/v), $1 \%$ sodium deoxycholate, $0.05 \%$ SDS, $1 \mathrm{mM}$ phenylmethyl sulfonyl fluoride). The lysates were centrifuged at $12,000 \mathrm{~g}$ for $10 \mathrm{~min}$ at $4{ }^{\circ} \mathrm{C}$ and the protein content in the supernatant was measured using the Lowry assay. Equal amounts of protein were separated on $10 \%$ SDS-PAGE and then transferred to nitrocellulose membranes (GE Healthcare, USA). After blocking with 5\% skim milk, the membranes were incubated with primary antibodies against Bax and Bcl-2 (1:500; Santa Cruz Biotechnology, USA), followed by the addition of horseradish peroxidase-conjugated secondary antibody. Protein bands were then developed with Western blot detection reagents (GE Healthcare). Beta-actin was included as an internal control.

In order to detect the release of cytochrome $C$ from mitochondria to the cytoplasm, L929 cells were washed with PBS and collected after exposure to $20 \mu \mathrm{M}$ DMAE-CB for $12 \mathrm{~h}$. The cells were resuspended in $1.5 \mathrm{~mL}$ cold MitoCyto Buffer (Applygen Biotechnology, China), lysed and centrifuged. The supernatant was then centrifuged again at $12,000 \mathrm{~g}$ for $10 \mathrm{~min}$ to separate the mitochondria (in pellet) and the cytoplasm (the supernatant). Western blot was performed for both cytoplasm protein and mitochondrial protein to study the possible release of cytochrome $\mathrm{C}$ from the mitochondria to the cytoplasm.

\section{Statistical analysis}

The result of the MTT assay was analyzed by one-way ANOVA followed by the post hoc Tukey test. The results of cell cycle analysis, annexin V/PI staining, ROS production and MMP measurement were analyzed by the nonparametric Mann-Whitney test. The difference between the experimental and control groups was considered to be significant when $\mathrm{P}<0.05$.

\section{Results}

\section{DMAE-CB-induced cell death in a dose-dependent manner}

DMAE-CB-induced cell death in L929 cells in a dosedependent manner. As shown in Figure 1A, 5 to $35 \mu \mathrm{M}$ DMAE-CB reduced the number of viable cells by approximately $30-80 \%$. Almost all cells were dead after exposure to DMAE-CB at concentrations higher than $40 \mu \mathrm{M}$. The $50 \%$ inhibition concentration $\left(\mathrm{IC}_{50}\right)$ for DMAE-CB was estimated to range from 25 to $35 \mu \mathrm{M}$. Figure 1B shows representative cell micrographs after treatment with various concentrations of DMAE-CB. While cells in the control group exhibited normal growth and typical polygonal appearance, many cells became round after treatment with $10 \mu \mathrm{M}$ DMAE-CB. The cell population density was further reduced by $20 \mu \mathrm{M}$ DMAE-CB and more cells showed a rounded shape. In the $40 \mu \mathrm{M}$ DMAE-CB group, almost no cell with normal appearance could be detected.

\section{DMAE-CB-induced cell cycle arrest}

A24-h exposure of $L 929$ cells to DMAE-CB induced G1and G2-phase arrest (Figure 2). Among control cells that received no treatment, about 58,6 , and $35 \%$ of cells were in the G1-, G2-, and S-phase of the cell cycle, respectively. Exposure to $10 \mu \mathrm{M}$ DMAE-CB had no significant influence on cell cycle progression. However, $20 \mu \mathrm{M}$ DMAE-CB significantly increased the percentage of cells residing in the G1- and G2-phase to 72 and $11 \%$, respectively, while at the same time reducing the percentage of cells residing in the S-phase to $17 \%$. Cell cycle analysis was not performed on cells after exposure to $40 \mu \mathrm{M}$ DMAE-CB because almost all cells were dead after treatment.

\section{DMAE-CB-induced apoptosis}

Apoptosis of L929 cells after 24 h of exposure to DMAECB was studied by annexin V/PI staining. Figure $3 A$ shows representative density blots of cells exposed to 10,20, 40 $\mu \mathrm{M}$ DMAE-CB while Figure 3B summarizes the results of flow cytometry analysis. Both apoptosis and necrosis were detected after DMAE-CB treatment. The percentage of early apoptotic cells peaked $(13.4 \%)$ when the monomer concentration was $20 \mu \mathrm{M}$ and then decreased to normal levels when the monomer concentration was further increased to $40 \mu \mathrm{M}$. On the other hand, a dose-dependent increase in the number of cells in late apoptosis/necrosis and necrosis was observed concomitant to a significant decrease in the number of viable cells.

\section{DMAE-CB-increased ROS production}

To determine if DMAE-CB-induced cytotoxicity is related to disturbance of redox balance, intracellular ROS production was monitored using the fluorometric probe DCFH-DA and flow cytometry analysis. Figure 4A and $\mathrm{C}$ are representative graphs of ROS production in L929 

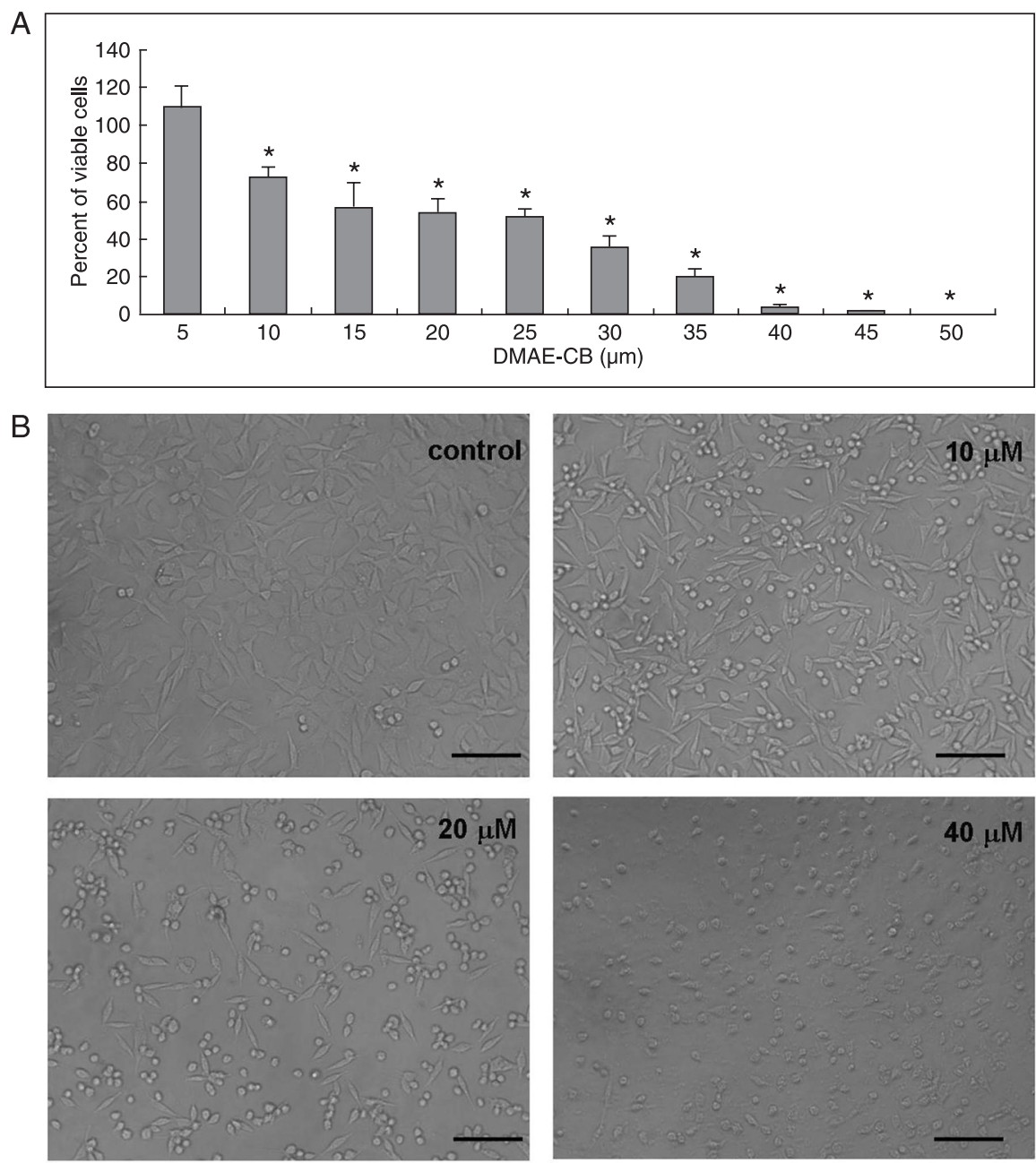

Figure 1. Cytotoxicity of methacryloxylethyl cetyl ammonium chloride (DMAE-CB) to L929 cells. A, DMAE-CB induced dose-dependent cell death in L929 cells as analyzed by the MTT assay $(N=10)$. Data are reported as means $\pm S D$. ${ }^{*} P<0.05$ compared to control (oneway ANOVA). $B$, Microscopic observation (100X) of L929 cells after exposure to DMAE-CB. In the presence of $10 \mu \mathrm{M}$ or higher concentrations of DMAE-CB, the cells grew poorly and exhibited reduced cell density and rounded or collapsed shape. Magnification bar $=50$ $\mu \mathrm{m}$.

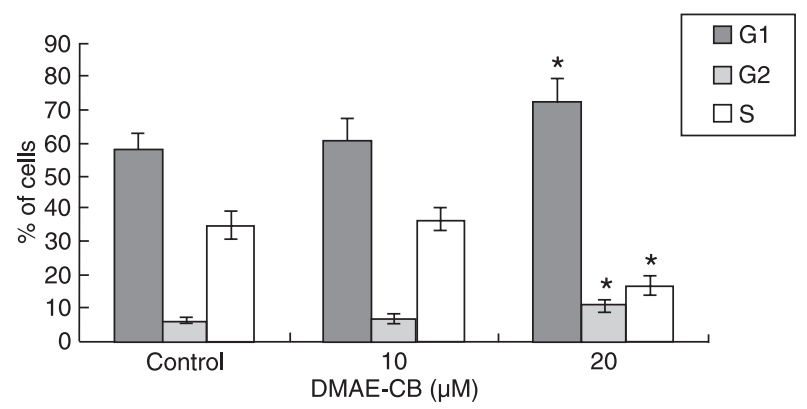

Figure 2. Induction of cell-cycle arrest by methacryloxylethyl cetyl ammonium chloride (DMAE-CB). After exposure to $20 \mu \mathrm{M}$ DMAE-CB, the percentage of cells in the G1- or G2-phase was significantly increased, while the percent of cells in the S-phase was reduced. Data are reported as means $\pm S D$. * $P<0.05$ compared to control (non-parametric Mann-Whitney test). 
cells after 1 or $6 \mathrm{~h}$ of antibacterial monomer treatment, respectively. A rapid increase of ROS production (rightward shift of the curve) was observed $1 \mathrm{~h}$ after treatment with
$40 \mu \mathrm{M}$ DMAE-CB. This overproduction of ROS was further enhanced after $6 \mathrm{~h}$. Figure $4 \mathrm{~B}$ and $\mathrm{D}$ summarize the results of four independent studies and exhibit a dose-dependent
A (F1)[A]BJX6.LMD:PMT2LOG/PMT3LOG

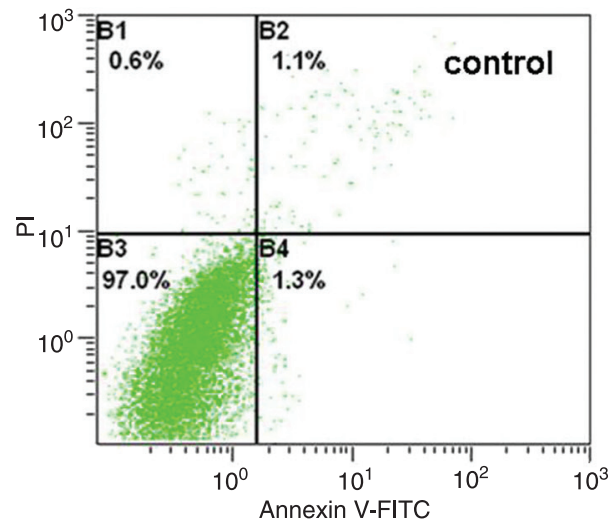

(F1)[A]BJX3Q.LMD:PMT2LOG/PMT3LOG

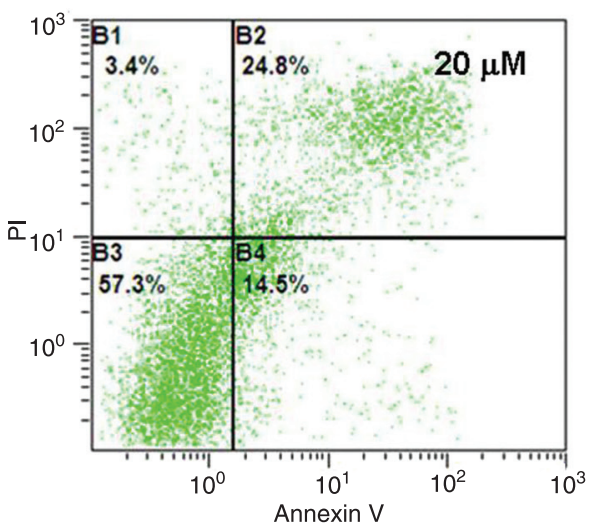

B

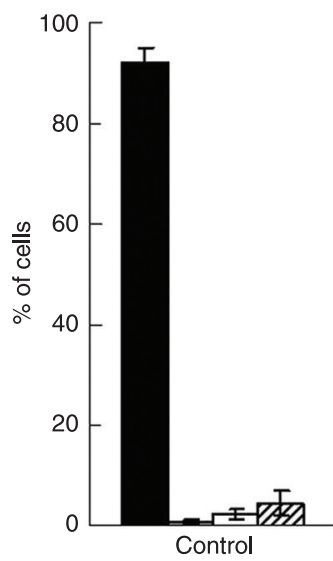

(F1)[A]YB3.LMD:PMT2LOG/PMT3LOG

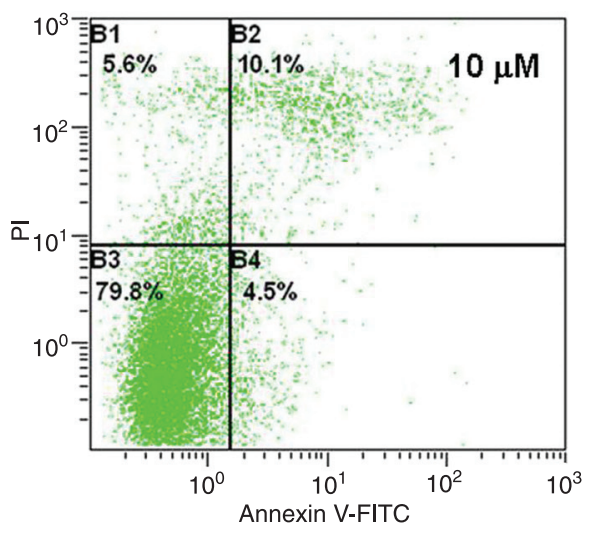

(F1)[A]YB6.LMD:PMT2LOG/PMT3LOG

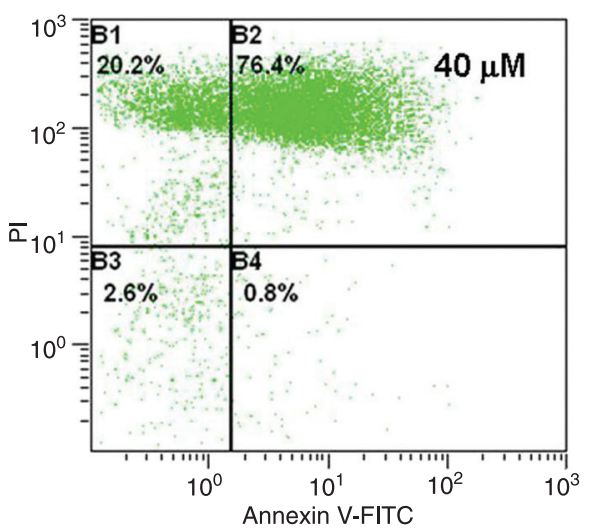

Annexin V-FITC

Figure 3. Induction of apoptosis in L929 cells by methacryloxylethyl cetyl ammonium chloride (DMAE-CB) as analyzed by annexin V/PI staining. A, Representative results are shown for cells treated with culture medium or 10, 20 , or $40 \mu \mathrm{M}$ DMAE-CB for $24 \mathrm{~h}$. The cell populations shown at lower left, lower right, upper right, and upper left represent living cells, early apoptotic cells, necrotic/late apoptotic cells, and necrotic cells, respectively. $B$, The results of four independent studies were summarized. The percentage of cells undergoing early apoptosis peaked in the $20 \mu \mathrm{M}$ DMEA-CB group. PI $=$ propidium iodide. Data are reported as means $\pm S D$. ${ }^{*} P$ $<0.05$ compared to respective control (non-parametric Mann-Whitney test). 
A

(F1)[A]BJX0.LMD:PMT2LOG

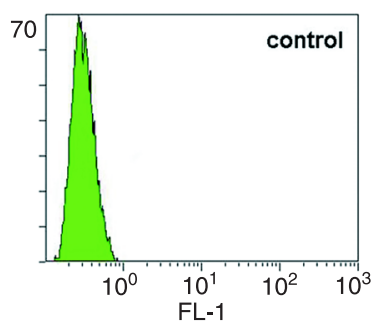

(F1)[A]BJXC6.LMD:PMT2LOG

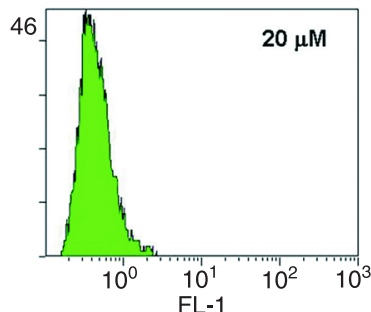

B

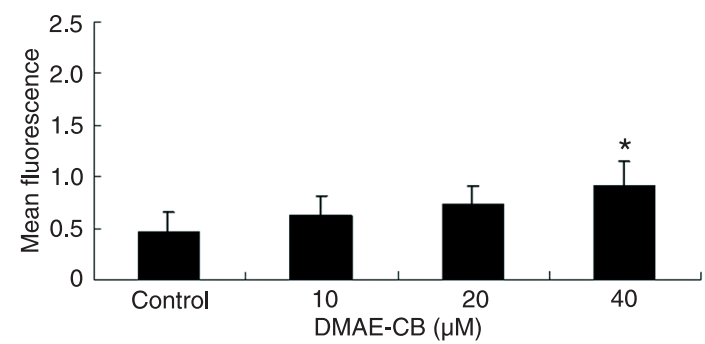

C

(F1)[A]BJXC2.LMD:PMT2LOG

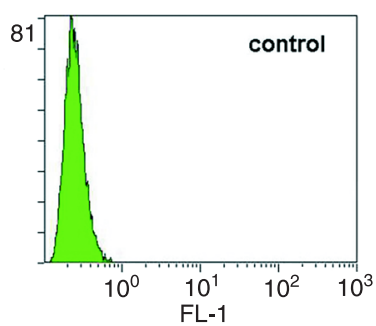

(F1)[A]BJXH2.LMD:PMT2LOG
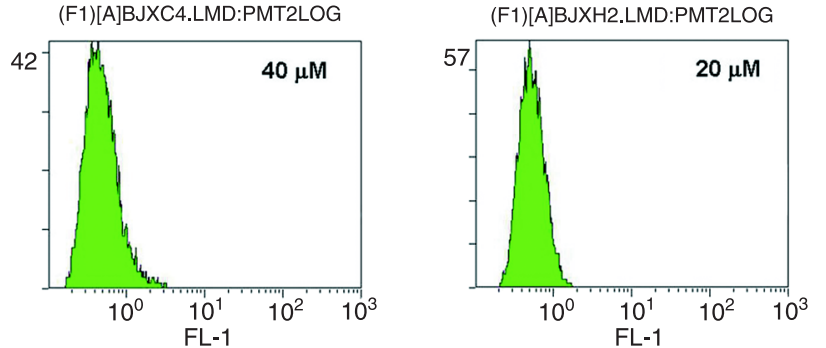

(F1)[A]BJXH4.LMD:PMT2LOG

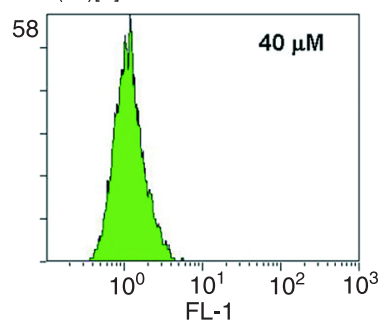

$\mathrm{D}$

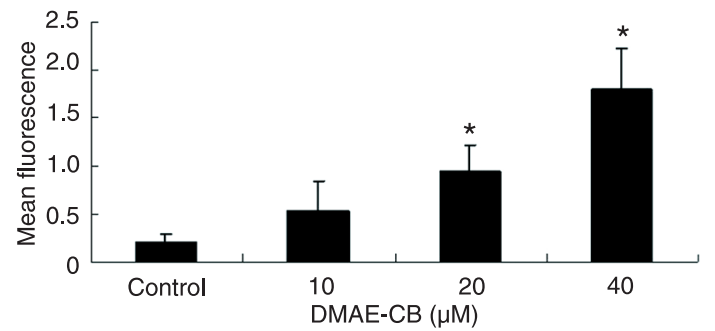

Figure 4. DMAE-CB induced ROS over-production. Representative graphs of flow cytometry analysis in cells exposed to DMAE-CB for $1(A)$ or $6(C)$ h are shown. A summary of four independent experiments indicates that intracellular ROS levels increased rapidly after $1 \mathrm{~h}(B)$ and this dose-dependent overproduction was further enhanced after $6 \mathrm{~h}(D)$. DMAE-CB = methacryloxylethyl cetyl ammonium chloride; ROS = reactive oxygen species. Data are reported as means $\pm \mathrm{SD}$. ${ }^{*} \mathrm{P}<0.05$ compared to control (non-parametric Mann-Whitney test).

increase in intracellular ROS level after DMAE-CB treatment. Maximum ROS production was observed in cells treated with $40 \mu \mathrm{M}$ DMAE-CB.

\section{DMAE-CB increased the percentage of cells with reduced MMP}

The loss of mitochondrial membrane potential is an important event in the intrinsic mitochondrial pathway of apoptosis (10). To determine whether this pathway was involved in DMAE-CB-related cytotoxicity, we treated the cells with DMAE-CB and determined their mitochondrial membrane potential using DiOC6 staining. As shown in Figure 5, a dose-dependent increase in the percentage of cells with reduced MMP was observed after treatment with DMAE-CB for $12 \mathrm{~h}$. Compared with control (5.3\%), the percentage of cells exhibiting reduced MMP after treatment of DMAE-CB at the concentration of 20 and $40 \mu \mathrm{M}$ was increased to 33.1 and $38.5 \%$, respectively. These results indicate that DMAE-CB-induced cell death is at lest partially related to the intrinsic mitochondrial apoptotic pathway.

\section{DMAE-CB interfered with Bcl-2 and Bax expression and induced cytochrome $C$ release}

Since the Bcl-2 family members play a critical role in mitochondrial-related apoptosis (11), Western blot analysis was performed at different times to assess the expression of the Bcl-2 family proteins, Bax and Bcl-2. The expression of Bax was up-regulated whereas the expression of $\mathrm{Bcl}-2$ was down-regulated with increasing time of exposure to $20 \mu \mathrm{M}$ DMAE-CB (Figure 6A). Western blot analysis indicated that cytochrome $\mathrm{C}$ was primarily localized in the mitochondria in the control group (Figure 6B). After the cells were treated with $20 \mu \mathrm{M}$ DMEA-CB for $12 \mathrm{~h}$, cytochrome $\mathrm{C}$ was also detected in the cytoplasm, while its amount in mitochondria was reduced. This observation indicated that cytochrome $C$ was released from the mitochondria into the cytoplasm after DMAE-CB treatment. 


\section{Discussion}

In this study, by using the well-established and stable mouse fibroblast cell line L929, we demonstrated that the antibacterial monomer DMAE-CB induced ROS production, cell cycle arrest, apoptosis, and necrosis in the cells tested. Furthermore, mitochondrial dysfunction and the mitochondria-related apoptotic pathway were involved in cell death induced by DMAE-CB.

The MTT assay revealed significant inhibitory effects of DMAE-CB on cell viability. The cytotoxicity of 2,2-bis(4(3-methacryloyloxy-2-hydroxypropoxy) phenyl)propane (Bis-GMA), 1,6-bis (methacryloxy-2-ethoxycarbonylamino)2,4,4-trimethylhexane (UDMA), TEGDMA, and HEMA against fibroblasts has been well documented (12). The ranking of the cytotoxicity of these commonly used monomers in terms of $\mathrm{IC}_{50}$ values after $24 \mathrm{~h}$ of exposure is Bis-GMA $(9.35 \mu \mathrm{M})>\operatorname{UDMA}(17.4 \mu \mathrm{M})>\operatorname{TEGDMA}(124.5$ $\mu \mathrm{M})$ > > HEMA $(468 \mu \mathrm{M})$. In the present study, the $\mathrm{IC}_{50}$ for DMAE-CB was estimated to be about 25 to $30 \mu \mathrm{M}$. This value was higher than that for Bis-GMA and UDMA, suggesting that the antibacterial monomer DMAE-CB is as safe as the basic dental monomer Bis-GMA that is routinely incorporated into various kinds of bonding systems and resin composites at high concentrations.

ROS are a group of highly reactive molecules that can readily damage biological molecules (13). Recent invetigations have indicated that the disturbance of cellular redox balance is involved in dental monomer-related cytotoxicity (14-17). However, to our knowledge, no report concerning the involvement of ROS overproduction in antibacterial monomer-related cytotoxicity has been published. Therefore, in the present study, we investigated the effect of an antibacterial monomer on the production of ROS. In agreement with previous reports about major resin monomers, we found that DMAE-CB induced a rapid overproduction of ROS in $L 929$ cells. Thus, we may speculate that the toxicity induced by DMAE-CB is correlated with a disturbance of redox balance. Whether antioxidants such as $\mathrm{N}$-acetyl-cysteine (NAC) and ascorbic acid could reduce the cytotoxicity of DMAE-CB is currently under further investigation.

ROS-induced DNA damage can subsequently cause cell cycle arrest or even apoptosis in cases of severe and irreversible damage $(18,19)$. In the present study, the percentage of cells in the S-phase was significantly reduced after exposure to high DMAE-CB concentrations, while the percentage of cells arrested in $\mathrm{G} 1$ or $\mathrm{G} 2$ was markedly increased to 72.1 and $10.6 \%$, respectively. This G1 and G2 cell cycle arrest induced by DMAE-CB can be attributed at least in part to the overproduction of ROS.

Oxidants, as well as a variety of other stimuli, can induce apoptosis $(20,21)$. In the present study, early apoptosis was observed in about $13.4 \%$ of cells after treatment with DMAE-CB at the concentration of $20 \mu \mathrm{M}$. Compared to published data about apoptosis induced by HEMA
(22), TEGDMA (23), and Bis-GMA (24), the percentage of cells undergoing apoptosis after DMAE-CB treatment was much lower. Although the possibility that different cells may have different sensitivity to apoptosis cannot be ruled out, some other explanation may also be valid. Owing to its positive charge (25), the cationic antibacterial monomer DMAE-CB, unlike other resin monomers, can easily adhere to the cell surface and alter the fluidity of the membrane, thereby disrupting its integrity and caus-

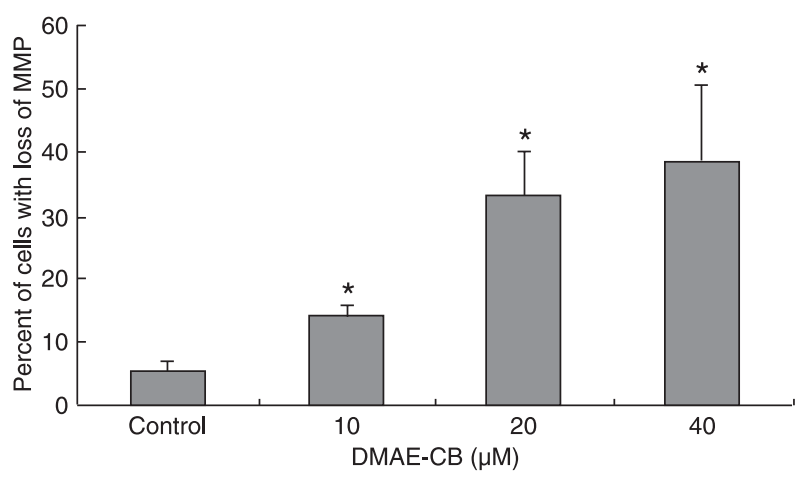

Figure 5. Methacryloxylethyl cetyl ammonium chloride (DMAE$\mathrm{CB}$ ) induced mitochondria dysfunction as indicated by the loss of mitochondrial membrane potential (MMP). Data are reported as means $\pm S D$ of four independent experiments. ${ }^{*} P<0.05 \mathrm{com}-$ pared to control (non-parametric Mann-Whitney test).
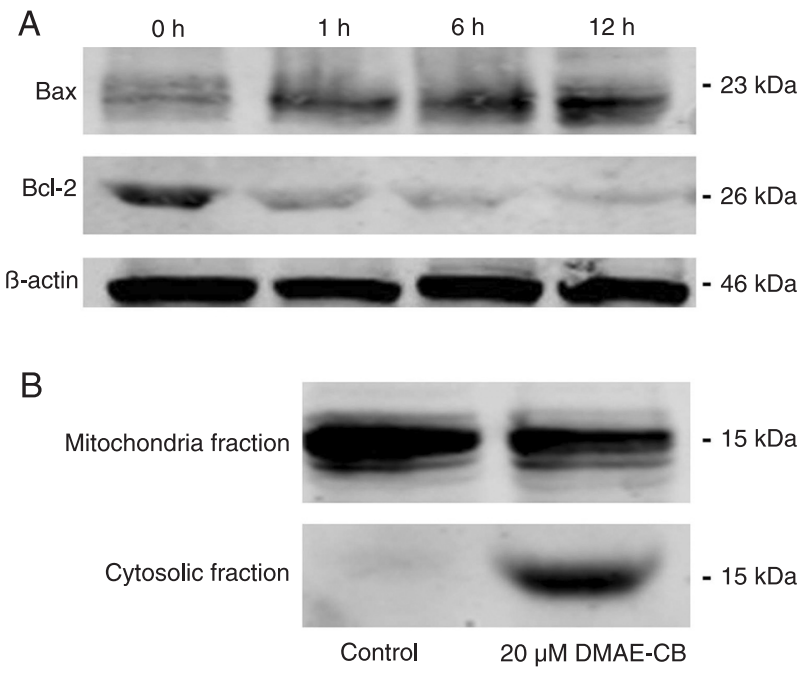

Figure 6. Western blot analysis of Bax and Bcl-2 expression, and cytochrome $\mathrm{C}$ release. A time-dependent shift of $\mathrm{Bax}$ and $\mathrm{Bcl}-2$ expression can be observed in L929 cells after exposure to 20 $\mu \mathrm{M}$ methacryloxylethyl cetyl ammonium chloride (DMAE-CB) $(A)$. The up-regulation of Bax was accompanied by a down-regulation of $\mathrm{Bcl}-2$. Moreover, a remarkable release of cytochrome $\mathrm{C}$ from the mitochondria to the cytosol was observed after 12-h treatment with $20 \mu \mathrm{M}$ DMAE-CB $(B)$. 
ing the cell to burst and undergo necrosis (9). This may be a possible reason for the relatively low apoptotic rate induced by the cationic antibacterial monomer DMAE-CB observed in this study.

It is of vital importance to investigate in detail the apoptosis induced by the antibacterial monomer DMAE-CB. Mitochondria, which are not only the main source of ROS but also an important target for the damaging effects of ROS (26), are a vital component of the intrinsic apoptotic pathway. Thus, in this study, we focused on the influence of DMAE-CB on the intrinsic mitochondrial apoptotic pathway. Similar to the study by Lefeuvre et al. (27) indicating that mitochondria damage was involved in the cytotoxic effect of TEGDMA (28), we found that the antibacterial monomer induced significant collapse of the mitochondrial membrane in L929 cells. Further Western blot analysis revealed that this loss of mitochondrial membrane potential was accompanied by the release of cytochrome $\mathrm{C}$. Moreover, the involvement of the mitochondrium-related intrinsic apoptosis pathway was reconfirmed by the time-dependent disturbance of the expression of Bax (a pro-apoptotic protein that accelerates the opening of the mitochondrial membrane pore) and $\mathrm{Bcl}-2$ (anti-apoptotic protein, which prevents the opening

\section{References}

1. De Munck J, Van Landuyt K, Peumans M, Poitevin A, Lambrechts $P$, Braem $M$, et al. A critical review of the durability of adhesion to tooth tissue: methods and results. J Dent Res 2005; 84: 118-132.

2. Imazato S. Bio-active restorative materials with antibacterial effects: new dimension of innovation in restorative dentistry. Dent Mater J 2009; 28: 11-19.

3. Li F, Chai ZG, Sun MN, Wang F, Ma S, Zhang L, et al. Antibiofilm effect of dental adhesive with cationic monomer. $J$ Dent Res 2009; 88: 372-376.

4. Xiao YH, Ma S, Chen JH, Chai ZG, Li F, Wang YJ. Antibacterial activity and bonding ability of an adhesive incorporating an antibacterial monomer DMAE-CB. J Biomed Mater Res B Appl Biomater 2009; 90: 813-817.

5. Li F, Chen J, Chai Z, Zhang L, Xiao Y, Fang M, et al. Effects of a dental adhesive incorporating antibacterial monomer on the growth, adherence and membrane integrity of Streptococcus mutans. J Dent 2009; 37: 289-296.

6. Schweikl H, Spagnuolo G, Schmalz G. Genetic and cellular toxicology of dental resin monomers. J Dent Res 2006; 85: 870-877.

7. Hume WR, Gerzina TM. Bioavailability of components of resin-based materials which are applied to teeth. Crit Rev Oral Biol Med 1996; 7: 172-179.

8. Noda M, Wataha JC, Kaga M, Lockwood PE, Volkmann KR, Sano $\mathrm{H}$. Components of dentinal adhesives modulate heat shock protein 72 expression in heat-stressed THP-1 human monocytes at sublethal concentrations. J Dent Res 2002; 81: $265-269$.

9. Nishida M, Imazato S, Takahashi $\mathrm{Y}$, Ebisu S, Ishimoto T, Nakano T, et al. The influence of the antibacterial monomer of the mitochondrial membrane pore) (28). All of these lines of evidence indicate that the mitochondrium-related apoptotic pathway plays an important role in DMAE-CBrelated cytotoxicity.

The antibacterial monomer DMAE-CB elicited cell-cycle arrest, necrosis and apoptosis in L929 cells in a dose-dependent manner. These detrimental effects were accompanied by the overproduction of ROS. This overproduction of ROS may initiate the apoptotic pathway by disturbing the expression of Bcl-2 super-family proteins, inducing collapse of mitochondrial membrane potential and subsequent release of cytochrome $\mathrm{C}$. Future research should be focused on the possible protective effect of antioxidants, such as NAC and ascorbic acid, on the cytotoxicity of DMAE-CB, and more detailed elucidation of the mechanism of apoptosis induced by this antibacterial monomer.

\section{Acknowledgments}

Research supported by the National Natural Science Foundation of China (\#81070861, \#81130078, and \#30901784).

12-methacryloyloxydodecylpyridinium bromide on the proliferation, differentiation and mineralization of odontoblast-like cells. Biomaterials 2010; 31: 1518-1532.

10. Gupta S, Kass GE, Szegezdi E, Joseph B. The mitochondrial death pathway: a promising therapeutic target in diseases. J Cell Mol Med 2009; 13: 1004-1033.

11. Kroemer $\mathrm{G}$. The proto-oncogene $\mathrm{Bcl}-2$ and its role in regulating apoptosis. Nat Med 1997; 3: 614-620.

12. Ratanasathien S, Wataha JC, Hanks CT, Dennison JB. Cytotoxic interactive effects of dentin bonding components on mouse fibroblasts. J Dent Res 1995; 74: 1602-1606.

13. Sturrock JE, Nunn JF. Chromosomal damage and mutations after exposure of Chinese hamster cells to high concentrations of oxygen. Mutat Res 1978; 57: 27-33.

14. Chang MC, Lin LD, Chan CP, Chang HH, Chen LI, Lin HJ, et al. The effect of BisGMA on cyclooxygenase-2 expression, PGE2 production and cytotoxicity via reactive oxygen species- and MEK/ERK-dependent and -independent pathways. Biomaterials 2009; 30: 4070-4077.

15. Chang $\mathrm{HH}$, Guo MK, Kasten FH, Chang MC, Huang GF, Wang YL, et al. Stimulation of glutathione depletion, ROS production and cell cycle arrest of dental pulp cells and gingival epithelial cells by HEMA. Biomaterials 2005; 26: 745-753.

16. Stanislawski L, Lefeuvre M, Bourd K, Soheili-Majd E, Goldberg $\mathrm{M}$, Perianin A. TEGDMA-induced toxicity in human fibroblasts is associated with early and drastic glutathione depletion with subsequent production of oxygen reactive species. J Biomed Mater Res A 2003; 66: 476-482.

17. Chang HH, Chang MC, Lin LD, Lee JJ, Wang TM, Huang $\mathrm{CH}$, et al. The mechanisms of cytotoxicity of urethane di- 
methacrylate to Chinese hamster ovary cells. Biomaterials 2010; 31: 6917-6925.

18. Shackelford RE, Kaufmann WK, Paules RS. Oxidative stress and cell cycle checkpoint function. Free Radic Biol Med 2000; 28: 1387-1404.

19. Shackelford RE, Innes CL, Sieber SO, Heinloth AN, Leadon SA, Paules RS. The Ataxia telangiectasia gene product is required for oxidative stress-induced $\mathrm{G} 1$ and $\mathrm{G} 2$ checkpoint function in human fibroblasts. J Biol Chem 2001; 276: 21951-21959.

20. Boonstra J, Post JA. Molecular events associated with reactive oxygen species and cell cycle progression in mammalian cells. Gene 2004; 337: 1-13.

21. Ryter SW, Kim HP, Hoetzel A, Park JW, Nakahira K, Wang X, et al. Mechanisms of cell death in oxidative stress. Antioxid Redox Signal 2007; 9: 49-89.

22. Paranjpe A, Bordador LC, Wang MY, Hume WR, Jewett A. Resin monomer 2-hydroxyethyl methacrylate (HEMA) is a potent inducer of apoptotic cell death in human and mouse cells. J Dent Res 2005; 84: 172-177.
23. Janke V, von Neuhoff N, Schlegelberger B, Leyhausen G, Geurtsen W. TEGDMA causes apoptosis in primary human gingival fibroblasts. J Dent Res 2003; 82: 814-818.

24. Engelmann J, Janke V, Volk J, Leyhausen G, von Neuhoff $\mathrm{N}$, Schlegelberger B, et al. Effects of BisGMA on glutathione metabolism and apoptosis in human gingival fibroblasts in vitro. Biomaterials 2004; 25: 4573-4580.

25. Marcotte L, Barbeau J, Lafleur M. Permeability and thermodynamics study of quaternary ammonium surfactantsphosphocholine vesicle system. J Colloid Interface Sci 2005; 292: 219-227.

26. Ott M, Gogvadze V, Orrenius S, Zhivotovsky B. Mitochondria, oxidative stress and cell death. Apoptosis 2007; 12: 913-922.

27. Lefeuvre M, Amjaad W, Goldberg M, Stanislawski L. TEGDMA induces mitochondrial damage and oxidative stress in human gingival fibroblasts. Biomaterials 2005; 26: 51305137.

28. Kelekar A, Thompson $\mathrm{CB}$. Bcl-2-family proteins: the role of the BH3 domain in apoptosis. Trends Cell Biol 1998; 8: 324-330. 